\title{
Investigating Fault Localization Techniques from Other Disciplines for Software Engineering
}

\author{
Árpád Beszédes \\ Department of Software Engineering, University of Szeged \\ Árpád tér 2., H-6720 Szeged, Hungary \\ beszedes@inf.u-szeged.hu
}

Keywords: Faults, Defects, Fault Localization, Software Fault Localization, Literature Review, Interdisciplinary Aspects.

Abstract: In many different engineering fields, fault localization means narrowing down the cause of a failure to a small number of suspicious components of the system. This activity is an important concern in many areas, and there have been a large number of techniques proposed to aid this activity. Some of the basic ideas used are common to different fields, but generally quite diverse approaches are applied. Our long-term goal with the presented research is to identify potential techniques from non-software domains that have not yet been fully leveraged to software faults, and investigate their applicability and adaptation to our field. We performed an analysis of related literature, not limiting the search to any specific engineering field, with the aim to find solutions in nonsoftware areas that could be most successfully adapted to software fault localization. We found out that few areas have significant literature in the topic that are good candidates for adaptation (computer networks, for instance), and that although some classes of methods are less suitable, there are useful ideas in almost all fields that could potentially be reused. As an example of potential novel techniques for software fault localization, we present three concrete techniques from other fields and how they could potentially be adapted.

\section{INTRODUCTION}

Failures in complex systems can cause damage to the environment, people's health and lives, or the operation of businesses and governments. Hence, possible underlying faults in the respective engineering disciplines are a high priority concern.

These complex systems may be mechanical, electrical, software-driven, or any combination thereof, but there is one common subtopic, the central theme of this article, fault localization. In a very general sense, fault localization in complex (software and non-software) systems means identifying components (parts, modules, software code parts, etc.) of the system that are responsible for a specific observed failure or set of failures. Note, that fault localization is closely related to the notion of fault detection, the main difference being that the latter merely shows that there is a fault somewhere in the system but does not tell where. ${ }^{1}$

Due to the importance of fault localization, a number of different discipline- and domain-specific so-

\footnotetext{
${ }^{1}$ In related literature, there is often a confusion about these terms and their exact meaning might be different accross various disciplines.
}

lutions have been proposed to aid this process in an automatic or semi-automatic manner. In some situations, fault detection and localization are not separately handled, but our goal with the present work was to concentrate on the fault localization aspects only.

In this work, our main concern are software faults. In particular, our aim is to find out whether there are techniques used in non-software domains that have not yet been considered for software faults but could be adapted to the same. We deal with (semi)automatic fault localization techniques from various engineering disciplines, and start with an interdisciplinary analysis of the topic. To this end, we performed a systematic literature review with a very specific goal, to discover their applicability to software fault localization. We then elaborate on the possible enhancements to existing techniques or devising novel approaches that were successful in other disciplines but has not yet been considered for software.

In any of the mentioned engineering areas, systems tend to be large and complex, and they are often connected to each other, forming even more complex systems-of-systems. Consequently, it may be very difficult to localize the origin (root cause) of occurring failures. Hence, various fields have developed 
approaches to automate the fault localization process. Naturally, each field deals with its peculiarities and many of the techniques are domain-dependent, yet we found out that there are some similarities across disciplines. Furthermore, some of the methods are generic and could be applied, theoretically, to any engineering field and fault localization problem.

Software fault localization has a large literature covering many different subtopics; see (Wong et al., 2016; Parmar and Patel, 2016). However, other areas, for instance, aerospace or electronics, have much longer histories and hence might provide ideas for advancing the software field. Next, a lot of research has been performed to design effective software fault localization algorithms and propose their use in different phases of the software process, most notably debugging. But, related research suggests that the practical applicability of research results in this area is still limited (Kochhar et al., 2016), and further research is needed to achieve more widespread use of automatic software fault localization by practitioners. We also noticed that recent results in this area are able to only marginally improve the effectiveness of previous techniques because it is increasingly difficult to make significant and highly novel contributions.

Hence the motivation for the present work; to investigate other engineering fields and find out if they employ techniques that could be adapted to software and thus advance this field. We found that in some cases there are barriers to the adoption of such techniques due to the fundamental differences in these (software and non-software) systems. Some of them arise from the fact that software systems are much more intangible, but also there are notable differences in how they are described and handled, e.g., types of behavioral models used to describe the expected behavior of the systems. In other cases, however, the techniques or some underlying ideas could be successfully adapted to software.

In previous work (Beszédes, 2019), we presented details of an interdisciplinary survey of fault localization techniques to aid software engineering. After providing the necessary background information and detailed assessment criteria, we presented the categorization of the identified methods according to several major fields. Also, a set of possible relationships between the areas has been presented. In the present work, we introduce three concrete examples of how techniques successfully used in other fields could potentially be adapted to software faults. Our aim with this approach is to illustrate the relevance of this research, but further details and more investigation are needed to elaborate on the actual implementability of the methods.
The paper is organized as follows. Section 2 describes the approach we used for the literature survey. In Section 3, we present the results of the literature analysis with our initial evaluation and example adoptable techniques. Finally, Section 4 concludes.

\section{LITERATURE SURVEY}

In this section, we briefly overview the process we followed in reviewing fault localization literature in various engineering fields.

In the first phase, we identified the most relevant papers with the help of the google, google scholar, ResearchGate, Mendeley and Scopus systems. We used a combination of generic search terms like "fault localization" and specific keywords that we expected to be relevant for our search: networks, electronics, engineering, operations, systems, etc. We also applied different variations and synonyms to these terms, which included localizing faults, failure diagnosis, problem diagnosis, error localization, and similar. This way, we were able to identify related works in many different engineering fields.

We restricted the search results to full-text scientific publications. We aimed at limiting the results to publications that appeared in peer-reviewed journals or conferences, however there were few exceptions such as doctoral theses and technical reports. The next filtering we applied was to limit the list to papers that correspond to some of the following categories: software-related, generic algorithms, and methods from loosely related engineering fields. For example, pure mathematical methods and approaches in non-related scientific branches like biomedicine, navigation, linguistics or other, were removed.

In addition to the repository and proceeding searches, we also performed lightweight snowballing (Wohlin, 2014) to mitigate the risk of omitting relevant literature. Finally, we consolidated the results by organizing the works by specific research groups or authors and concentrating on a few relevant reports by the same team.

We then performed an initial classification of the papers based on what fundamental area they belong to. We used a simple classification in this respect: software, networking, other engineering and various/generic. There exists a large amount of publications that deal with fault localization in computer networks, hence we established a separate category for this area. The other engineering category includes all methods that belong to a specific engineering field other than software or networks. Finally, there are some approaches that are not limited to any specific 
field (although some of them include one or more example applications); in this sense, they are generic. We used the same category to denote methods belonging to some other various fields.

Finally, we extended the classification using other criteria which might be additionally useful to determine the methods' usability in the software domain. These criteria included:

- The basic approach on which the method is based on: machine learning, statistical analysis, entropy-based, etc.

- Single or multiple faults are handled.

- Basic type of data the method relies on.

- The need for a behavior model.

- Other properties of the related studies such as kind of empirical measurements, size of data sets, and availability of data or implementation.

Details about these criteria and the analysis outcomes are omitted from this paper due to space considerations, but all the data are available in previous work (Beszédes, 2019).

\section{ANALYSIS AND PROPOSED NEW TECHNIQUES}

Our initial findings indicate that it is not easy to pinpoint only a few candidate methods, rather many of them may provide interesting ideas, even if not the complete method is adapted.

Below, we provide an overview of the most notable techniques we identified and their initial evaluation for each of the main fundamental areas. Software is intentionally omitted from this list, as we wanted to find approaches not yet existing in our field. For each of the three relevant categories (Networking, Other Engineering and Generic Methods), we propose specific new software fault localization methods based on the related approaches. These can serve as examples of interdisciplinary application of a technique, but this does not necessarily mean that we cannot benefit from other, less directly related methods.

\subsection{Networking}

The main representative literature we identified in the networking category are: (Steinder and Sethi, 2004c), (Alekseev and Sayenko, 2014), (Yu et al., 2010), (Brodie et al., 2002), (Brodie et al., 2003), (Chao et al., 1999), (Chen et al., 2004), (Cheng et al., 2010), (Deng et al., 1993), (Fecko and Steinder, 2001), (Lu et al., 2013a), (Hood and Ji, 1997), (Kant et al.,
2002), (Kant et al., 2004), (Katzela and Schwartz, 1995), (Kompella et al., 2005), (Lu et al., 2013b), (Mohamed, 2017), (Natu and Sethi, 2005), (Natu and Sethi, 2006), (Natu and Sethi, 2007a), (Natu and Sethi, 2007b), (Rish et al., 2004), (Tang et al., 2005), (Traczyk, 2004), (Wang et al., 2012), (Zhang et al., 2011), (Boubour et al., 1997), (Aghasaryan et al., 1997), (Aghasaryan et al., 1998), (Steinder and Sethi, 2004a), (Steinder and Sethi, 2004b), (BenHaim, 1980).

A common element of network fault localization is the use of probabilistic approaches (such as conditional probabilities and Bayes networks) (Chao et al., 1999; Tang et al., 2005; Steinder and Sethi, 2004b), and machine learning (Chen et al., 2004; Deng et al., 1993; Hood and Ji, 1997), which could probably be adapted to software faults.

The probing method in computer networks, e. g. (Steinder and Sethi, 2004c), is an almost direct analogy to software fault localization (a probe is a program that executes on a particular network node and sends commands or transactions to the other elements of the network, and the responses are observed and their various properties are measured). A network node may correspond to a software component, a probe can be seen as a test case, and the responses from the network can be identified as the dynamic behavior of the system by executing the test cases.

In this case, the Spectrum-Based Fault Localization class of methods (Abreu et al., 2009a; Parmar and Patel, 2016; Wong et al., 2016) could be naturally extended using results from networking. Here, the basis of the algorithms is the so-called program spectrum, which in its basic form is essentially a binary matrix consisting of the test cases in its rows and the program elements in the columns. A 1 in a matrix element represents that the test case executes the corresponding code element. Then, based on the test case outcomes, various statistics are computed for each program element about the number of failing and passing test case executions traversing it, and the most suspicious code elements are reported to the user (many failing test cases and few passing ones make a code element more suspicious).

Approaches by Yu et al. (Yu et al., 2010), Brodie et al. (Brodie et al., 2002; Brodie et al., 2003), Cheng et al. (Cheng et al., 2010), Natu et al. (Natu and Sethi, 2005; Natu and Sethi, 2006; Natu and Sethi, 2007a; Natu and Sethi, 2007b) provide various optimizations to the basic probing approaches, which are good candidates for adaptation to (spectrum-based) software fault localization.

In particular, the following can be an optimized approach to fault localization based on the results 
from publications in the networking field. These steps could be for optimal test suite construction (here, a test case corresponds to a probe in networking):

1. Start from an initial test case set, e. g., optimizing for high code coverage.

2. Determining the diagnosis ability of this set. This can be based on some property using entropy or some other measurement. Similar results are already available in software fault localization (Perez et al., 2017; Perez and Abreu, 2018).

3. Finding the minimal set of test cases, based on some heuristics such as greedy search.

Based on the mentioned works, the program spectra (coverage matrix) can be extended to a Bayesian network that encodes probabilistic dependencies between the possible faults (causes) and the test outcomes (symptoms) (Brodie et al., 2002).

\subsection{Other Engineering Fields}

We identified the engineering fields of aerospace and (power) electronics that could potentially be the most useful for our purposes, along with a few additional techniques from specific disciplines like oil pipelines and chemistry. For these fields, our main findigs are: aerospace (Adamovits and Pagurek, 1993; Balaban et al., 2007; W. Dries, 1990), power electronics (Benbouzid et al., 1999; Beschta et al., 1993; Poon, 2015; Tanwani et al., 2011), electronics (Peischl and Wotawa, 2006), oil pipelines (Digernes, 1980), and chemistry (Venkatasubramanian et al., 1990).

The most promising novel approach to software fault localization is to use behavioral software models against which the actual behavior could be compared to. A behavioral model essentially describes the expected functionality of the system which might be expressed using various formalisms, either graphical or textual. This area is often refferred to as ModelBased Diagnosis. This concept has not yet been investigated in depth for our field. The closest approach is Abreu et al.'s work on using Bayesian framework for software fault localization (Abreu et al., 2009b), however this technique does not incorporate other components of model-based diagnosis overviewed below.

In the aerospace industry, the use of model based reasoning, a branch of artificial intelligence, is frequent (Adamovits and Pagurek, 1993; Balaban et al., 2007; W. Dries, 1990). In power electronics (Poon, 2015; Benbouzid et al., 1999; Beschta et al., 1993), the methods also frequently utilize various models describing the system. Although somewhat different, but some approaches to fault localization in electronic circuits are using behavioral models combined with simulation. In addition, in some cases the description of the hardware is done in a similar way to software source code (Peischl and Wotawa, 2006), which makes it possibly more easier to adapt to our field. Other areas we investigated often use simulation and probabilistic approaches as well (Digernes, 1980), or machine learning with neural networks (Venkatasubramanian et al., 1990), and in these cases a model of the system is required as well. Often, advanced concepts are applied in these areas such as Kalman filters to increase the accuracy of fault estimates.

A model-based approach to fault localization in software requires reliable behavioral models that describe the expected behavior. This is then compared to the observed data from system under examination. Models are in some cases already available; especially, in the case of critical systems. Here, systematic and rigorous requirement elicitation, fault mode and effect analysis, and test design is done. Models and their languages depend on the domain and the modeling approach used. Although the reasoning components of the system may also include experimental knowledge, the model can largely be seen as a black box, which encodes the required behavior and is determined during early software engineering phases. For successful model-based diagnosis, models need to be accurate and independent of the reasoning structure. Completeness is also important, but the faulty behaviour is generally little-known. Models are often encoded in the form of a simulation.

Once the model is available, model-based approach to fault localization in software would follow the usual approach in model-based diagnosis: given observations of the system, the system is simulated using the model, and the observations actually made are compared to the observations predicted by the simulation. The following specific subtasks would be required in general: data acquisition (producing dynamic operational data from program executions, which can be performed using existing approaches such as profiling, logging and instrumentation), fault detection (performing the tests and comparison to the model), hypothesis generation and pruning (producing and analyzing "infection chains" (Zeller, 2005) from defects to the symptoms), and hypothesis validation (through formal model analysis or simulation).

\subsection{Generic Methods}

In the generic category, we identified the following main techniques: (Isermann, 1984), (Massoumnia et al., 1986), (Svärd, 2012), (Varga, 2003), (Lerner and Parr, 2000), (Olivier-Maget et al., 2009), (Shchekotykhin et al., 2016), (Mehra and Peschon, 
1971), (Bouloutas et al., 1993), (Frank, 1996), (Tidriri et al., 2016), (de Kleer, 2009), (de Kleer and Williams, 1987).

It is worthwhile to note that some techniques we listed here (e.g. Kleer et al. (de Kleer, 2009; de Kleer and Williams, 1987)) have their main application in the mentioned fields from previous sections such as electronic circuits and computer networks, and are based on techniques such as entropy minimization and probabilistic approach. In particular, Bayesian framework is the basis for Kleer et al.'s technique, which has also been reused for software (Abreu et al., 2009b).

Model-Based Diagnosis, mentioned in the previous section, aims at finding the fault of an observed system based on the knowledge about the system's expected behavior, which can be discussed in a generic, domain-independent manner (Shchekotykhin et al., 2016; Frank, 1996; de Kleer, 2009; de Kleer and Williams, 1987). Many of these model the system as a process, and hence process analysis approaches are used from control theory (Isermann, 1984; Massoumnia et al., 1986; Svärd, 2012; Venkatasubramanian et al., 1990; Mehra and Peschon, 1971).

For software fault localization, we propose to further investigate the combination of model based approaches with data driven methods (which process a large amount of data from the systems output and are based on training data for a correctly working system). Tidridi et al. (Tidriri et al., 2016) present an overview of the approach. We believe this could be a promising way because a reliable and complete model is often not available for software, but the operational data from software executions is easily obtainable, e. g. from logs. Tidridi et al. also refer other related works in this area, which can be useful sources for more information about this set of techniques.

In particular, in a data driven approach, a large amount of operational data (from logs, etc) are collected in which the fault is first detected (whether the system behavior matches the expected one) and then classified (determining the type of the fault). Typical classification types are supervised approaches based on manually prepared training data (e. g., using Neural Networks), and unsupervised methods such as various statistical techniques like Principal Component Analysis. These techniques can help model-based diagnosis is various ways, because in practice it is very difficult to develop accurate mathematical models for large and complex software systems. For example, the primary form of behavior differences to be observed by a model-based approach (see the previous section) can be generated and learned from a large amount of dynamic operational data.

\section{CONCLUSIONS}

The purpose of the presented work was to demonstrate that there is a lot of potential in interdisciplinary application of fault localization techniques from several different engineering fields to software faults. We performed a literature review involving various fields, computer networks, aerospace, (power) electronics, etc., and found that although in many cases the too large differences in the domains permit the techniques' application to our field, there are several ideas that could be leveraged for software.

Preliminary results presented in preceding sections could provide a starting point for additional analysis of the techniques, and the selection of the most promising approaches for further consideration. We also present three concrete approaches and how they could be adapted to software fault localization.

Although we performed the literature analysis in a systematic way, we cannot claim its completeness. Indeed, we did not dive into the details of any particular subfield or set of techniques. Instead, we concentrated on locating the most important approaches from various fields. Nevertheless, we believe that the survey in its present state is suitable for us to start designing in detail novel approaches to software fault localization, and for other readers to obtain a wider view of this important and diverse topic.

As future work, we will continue searching for other related techniques, extend the assessment with additional aspects and evaluate the most promising approaches in more detail. We will eventually start implementing the new techniques for software fault localization and compare their performance to other, already established techniques in this field.

Detailed assessment data of the literature survey are available from the author.

\section{ACKNOWLEDGEMENTS}

Árpád Beszédes was supported by the János Bolyai Research Scholarship of the Hungarian Academy of Sciences. The author would like to thank the supporting work of Ottó Eötvös and Brúnó Ilovai who were supported by project EFOP-3.6.3-VEKOP-162017-0002, co-funded by the European Social Fund. Ministry of Human Capacities, Hungary grant 203913/2018/FEKUSTRAT is acknowledged. 


\section{REFERENCES}

Abreu, R., Zoeteweij, P., and v. Gemund, A. J. C. (2009a). Spectrum-based multiple fault localization. In 2009 IEEE/ACM International Conference on Automated Software Engineering, pages 88-99.

Abreu, R., Zoeteweij, P., and Van Gemund, A. J. C. (2009b). A new bayesian approach to multiple intermittent fault diagnosis. In Proceedings of the 21st International Jont Conference on Artifical Intelligence, IJCAI'09, pages 653-658.

Adamovits, P. J. and Pagurek, B. (1993). Simulation (model) based fault detection and diagnosis of a spacecraft electrical power system. In Proceedings of 9th IEEE Conference on Artificial Intelligence for Applications, pages 422-428.

Aghasaryan, A., Fabre, E., Benveniste, A., Boubour, R., and Jard, C. (1997). A petri net approach to fault detection and diagnosis in distributed systems. ii. extending viterbi algorithm and hmm techniques to petri nets. In Proceedings of the 36th IEEE Conference on Decision and Control, volume 1, pages 726-731 vol.1.

Aghasaryan, A., Fabre, E., Benveniste, A., Boubour, R., and Jard, C. (1998). Fault detection and diagnosis in distributed systems: An approach by partially stochastic petri nets. Discrete Event Dynamic Systems, 8(2):203231.

Alekseev, D. and Sayenko, V. (2014). Proactive fault detection in computer networks. In 2014 First International Scientific-Practical Conference Problems of Infocommunications Science and Technology, pages 90-91.

Balaban, E., Narasimhan, S., Cannon, H. N., and Brownston, L. S. (2007). Model-based fault detection and diagnosis system for nasa mars subsurface drill prototype. In 2007 IEEE Aerospace Conference, pages $1-13$.

Ben-Haim, Y. (1980). An algorithm for failure location in a complex network. Nuclear Science and Engineering, 75(2):191-199.

Benbouzid, M. E. H., Vieira, M., and Theys, C. (1999). Induction motors' faults detection and localization using stator current advanced signal processing techniques. IEEE Transactions on Power Electronics, 14(1):1422.

Beschta, A., Dressler, O., Freitag, H., Montag, M., and Struss, P. (1993). Model-based approach to fault localization in power transmission networks. Intelligent Systems Engineering, 2:3 - 14

Beszédes, Á. (2019). Interdisciplinary survey of fault localization techniques to aid software engineering. Acta Polytechnica Hungarica, 16(3):207-226.

Boubour, R., Jard, C., Aghasaryan, A., Fabre, E., and Benveniste, A. (1997). A petri net approach to fault detection and diagnosis in distributed systems. i. application to telecommunication networks, motivations, and modelling. In Proceedings of the 36th IEEE Conference on Decision and Control, volume 1, pages 720725 vol.1.

Bouloutas, A. T., Hart, G. W., and Schwartz, M. (1993). Fault identification using a finite state machine model with unreliable partially observed data sequences. IEEE Transactions on Communications, 41(7):10741083.

Brodie, M., Rish, I., and Ma, S. (2002). Intelligent probing: A cost-effective approach to fault diagnosis in computer networks. IBM Systems Journal, 41(3):372-385.

Brodie, M., Rish, I., Ma, S., and Odintsova, N. (2003). Active probing strategies for problem diagnosis in distributed systems. In Proceedings of the 18th International Joint Conference on Artificial Intelligence, IJCAI'03, pages 1337-1338, San Francisco, CA, USA. Morgan Kaufmann Publishers Inc.

Chao, C. S., Yang, D. L., and Liu, A. C. (1999). An automated fault diagnosis system using hierarchical reasoning and alarm correlation. In Proceedings 1999 IEEE Workshop on Internet Applications (Cat. No.PR00197), pages 120-127.

Chen, M., Zheng, A. X., Lloyd, J., Jordan, M. I., and Brewer, E. (2004). Failure diagnosis using decision trees. In Proceedings of the International Conference on Autonomic Computing, pages 36-43.

Cheng, L., Qiu, X., Meng, L., Qiao, Y., and Boutaba, R. (2010). Efficient active probing for fault diagnosis in large scale and noisy networks. In 2010 Proceedings IEEE INFOCOM, pages 1-9.

de Kleer, J. (2009). Diagnosing multiple persistent and intermittent faults. In Proceedings of the Twenty-First International Joint Conference on Artificial Intelligence (IJCAI 2009), pages 733-738.

de Kleer, J. and Williams, B. C. (1987). Diagnosing multiple faults. Artificial Intelligence, 32(1):97 - 130.

Deng, R. H., Lazar, A. A., and Wang, W. (1993). A probabilistic approach to fault diagnosis in linear lightwave networks. IEEE Journal on Selected Areas in Communications, 11(9):1438-1448.

Digernes, T. (1980). Real-Time Failure-Detection and Identification Applied to Supervision of Oil Transport in Pipelines. Modeling, Identification and Control, 1(1):39-49.

Fecko, M. A. and Steinder, M. (2001). Combinatorial designs in multiple faults localization for battlefield networks. In 2001 MILCOM Proceedings Communications for Network-Centric Operations: Creating the Information Force (Cat. No.01CH37277), volume 2, pages 938-942 vol.2.

Frank, P. (1996). Analytical and qualitative model-based fault diagnosis : A survey and some new results. $E$ ropean Journal of Control, 2(1):6 - 28.

Hood, C. S. and Ji, C. (1997). Proactive network-fault detection. IEEE Transactions on Reliability, 46(3):333341.

Isermann, R. (1984). Process fault detection based on modeling and estimation methods: A survey. Automatica, 20(4):387 - 404

Kant, L., Chen, W., Lee, C.-W., Sethi, A., Natu, M., Luo, L., and Shen, C. (2004). D-flash : Dynamic fault localization and self-healing for battlefield networks. In Applied Soft Computing - ASC, pages 1-2.

Kant, L. A., Sethi, A. S., and Steinder, M. (2002). Fault localization and self-healing mechanisms for fcs net- 
works. In Proceedings of the 23rd Army Science Conference, pages 1-8.

Katzela, I. and Schwartz, M. (1995). Schemes for fault identification in communication networks. IEEE/ACM Trans. Netw., 3(6):753-764.

Kochhar, P. S., Xia, X., Lo, D., and Li, S. (2016). Practitioners' expectations on automated fault localization. In Proceedings of the 25th International Symposium on Software Testing and Analysis - ISSTA 2016, pages 165-176, New York, New York, USA. ACM Press.

Kompella, R. R., Yates, J., Greenberg, A., and Snoeren, A. C. (2005). Ip fault localization via risk modeling. In Proceedings of the $2 \mathrm{Nd}$ Conference on Symposium on Networked Systems Design \& Implementation - Volume 2, NSDI'05, pages 57-70, Berkeley, CA, USA. USENIX Association.

Lerner, U. and Parr, R. (2000). Bayesian fault detection and diagnosis in dynamic systems. In In Proc. AAAI, pages 531-537.

Lu, L., Xu, Z., Wang, W., and Sun, Y. (2013a). A new fault detection method for computer networks. Reliability Engineering \& System Safety, 114:4551.

Lu, L., Xu, Z., Wang, W., and Sun, Y. (2013b). A new fault detection method for computer networks. Reliability Engineering \& System Safety, 114(Supplement C):45 -51 .

Massoumnia, M.-A., Verghese, G. C., and Willsky, A. S. (1986). Failure detection and identification in linear time-invariant systems. Technical Report LIDS-P1578, MASSACHUSETTS INSTITUTE OF TECHNOLOGY.

Mehra, R. and Peschon, J. (1971). An innovations approach to fault detection and diagnosis in dynamic systems. Automatica, 7(5):637 - 640.

Mohamed, A. (2017). Fault Detection and Identification in Computer Networks: A Soft Computing Approach. $\mathrm{PhD}$ thesis, UWSpace.

Natu, M. and Sethi, A. S. (2005). Adaptive fault localization in mobile ad hoc battlefield networks. In MILCOM 2005 - 2005 IEEE Military Communications Conference, pages 814-820 Vol. 2.

Natu, M. and Sethi, A. S. (2006). Active probing approach for fault localization in computer networks. In 2006 4th IEEE/IFIP Workshop on End-to-End Monitoring Techniques and Services, pages 25-33.

Natu, M. and Sethi, A. S. (2007a). Efficient probing techniques for fault diagnosis. In Second International Conference on Internet Monitoring and Protection (ICIMP 2007), pages 20-20.

Natu, M. and Sethi, A. S. (2007b). Probabilistic fault diagnosis using adaptive probing. In Clemm, A., Granville, L. Z., and Stadler, R., editors, Managing Virtualization of Networks and Services, pages 38-49, Berlin, Heidelberg. Springer Berlin Heidelberg.

Olivier-Maget, N., Negny, S., Htreux, G., and Lann, J.M. L. (2009). Fault diagnosis and process monitoring through model-based and case based reasoning. Computer Aided Chemical Engineering, 26(Supplement C):345 - 350 .
Parmar, P. and Patel, M. (2016). Software fault localization: A survey. International Journal of Computer Applications, 154(9):6-13.

Peischl, B. and Wotawa, F. (2006). Automated source-level error localization in hardware designs. IEEE Design Test of Computers, 23(1):8-19.

Perez, A. and Abreu, R. (2018). Leveraging qualitative reasoning to improve sfl. In Proceedings of the TwentySeventh International Joint Conference on Artificial Intelligence, IJCAI-18, pages 1935-1941. International Joint Conferences on Artificial Intelligence Organization.

Perez, A., Abreu, R., and van Deursen, A. (2017). A testsuite diagnosability metric for spectrum-based fault localization approaches. In Proceedings of the 39th International Conference on Software Engineering, ICSE' 17, pages 654-664.

Poon, J. (2015). Model-based fault detection and identification for power electronics systems. Master's thesis, EECS Department, University of California, Berkeley.

Rish, I., Brodie, M., Odintsova, N., Ma, S., and Grabarnik, G. (2004). Real-time problem determination in distributed systems using active probing. In 2004 IEEE/IFIP Network Operations and Management Symposium (IEEE Cat. No.04CH37507), volume 1, pages 133-146 Vol.1.

Shchekotykhin, K., Schmitz, T., and Jannach, D. (2016). Efficient sequential model-based fault-localization with partial diagnoses. In Proceedings of the TwentyFifth International Joint Conference on Artificial Intelligence, IJCAI'16, pages 1251-1257. AAAI Press.

Steinder, M. and Sethi, A. (2004a). Probabilistic fault diagnosis in communication systems through incremental hypothesis updating. Computer Networks, 45(4):537 -562 .

Steinder, M. and Sethi, A. S. (2004b). Probabilistic fault localization in communication systems using belief networks. IEEE/ACM Transactions on Networking, 12(5):809-822.

Steinder, M. and Sethi, A. S. (2004c). A survey of fault localization techniques in computer networks. Science of Computer Programming, 53(2):165 - 194.

Svärd, C. (2012). Methods for Automated Design of Fault Detection and Isolation Systems with Automotive Applications. PhD thesis, Linköping University, Department of Electrical Engineering, Vehicular Systems.

Tang, Y., Al-Shaer, E. S., and Boutaba, R. (2005). Active integrated fault localization in communication networks. In 2005 9th IFIP/IEEE International Symposium on Integrated Network Management, 2005. IM 2005., pages 543-556. IEEE.

Tanwani, A., Dominguez-Garcia, A. D., and Liberzon, D. (2011). An inversion-based approach to fault detection and isolation in switching electrical networks. IEEE Transactions on Control Systems Technology, 19(5):1059-1074.

Tidriri, K., Chatti, N., Verron, S., and Tiplica, T. (2016). Bridging data-driven and model-based approaches for process fault diagnosis and health monitoring: A re- 
view of researches and future challenges. Annual Reviews in Control, 42:63 - 81 .

Traczyk, W. (2004). Probes for fault localization in computer networks. Journal of Telecommunications and Information Technology, nr 3:23-27.

Varga, A. (2003). On computing least order fault detectors using rational nullspace bases. IFAC Proceedings Volumes, 36(5):227 - 232. 5th IFAC Symposium on Fault Detection, Supervision and Safety of Technical Processes 2003, Washington DC, 9-11 June 1997.

Venkatasubramanian, V., Vaidyanathan, R., and Yamamoto, Y. (1990). Process fault detection and diagnosis using neural networks. i. steady-state processes. Computers \& Chemical Engineering, 14(7):699 - 712.

W. Dries, R. (1990). Model-based reasoning in the detection of satellite anomalies. Master's thesis, AIR FORCE INSTITUTE OF TECHNOLOGY.

Wang, B., Wei, W., Dinh, H., Zeng, W., and Pattipati, K. R. (2012). Fault localization using passive end-to-end measurements and sequential testing for wireless sensor networks. IEEE Transactions on Mobile Computing, 11(3):439-452.

Wohlin, C. (2014). Guidelines for snowballing in systematic literature studies and a replication in software engineering. In Proc. of Evaluation and Assessment in Software Engineering (EASE).

Wong, W. E., Gao, R., Li, Y., Abreu, R., and Wotawa, F. (2016). A survey on software fault localization. IEEE Trans. Softw. Eng., 42(8):707-740.

Yu, L., Qiu, X., Qiao, Y., Chen, X., and Liu, Y. (2010). Optimizing probe selection algorithms for fault localization. In 2010 3rd IEEE International Conference on Broadband Network and Multimedia Technology (IC-BNMT), pages 200-204.

Zeller, A. (2005). Why Programs Fail: A Guide to Systematic Debugging. Morgan Kaufmann Publishers Inc., San Francisco, CA, USA.

Zhang, X., Zhou, Z., Hasker, G., Perrig, A., and Gligor, V. (2011). Network fault localization with small tcb. In 2011 19th IEEE International Conference on Network Protocols, pages 143-154. 